\title{
Sizing in Phased Array Technique using Diffraction and Amplitude Displacement
}

\author{
G. Nardoni, M. Certo, P. Nardoni \\ I\&T Nardoni Institute
}

\begin{abstract}
This report shows the results of an experimental work carried out with the aim to improve defect analysis and sizing of both volumetric and planar defects, detected and imaged with the phased array probe technique. The phased array technique produces S-scan type images of defects which put easily in evidence the structure of the relevant echo indications. Depending of defect morphology, we can see diffracted echoes from planar defects, and also secondary echoes (improperly considered as diffracted echoes), generated by a complex conversion mode process, from volumetric ones. We can see also single indications, when defect height is comparable with ultrasonic wavelength or its complex geometry destroy diffracted or secondary indications, and, finally, we can see clustered indications. Thus we have to recognize such different image structures in order to apply the most suitable sizing procedure. The obtained results are very satisfactory and allow us to be more confident in apply phased array technique.
\end{abstract}

\section{Introduction}

Current phased array technique allows us to perform ultrasonic inspection with production of real time images (specifically sectorial scan type images) whose structure depends on defect geometry and its orientation with respect to ultrasonic probe. Such images show features that in standard fixed angle probe technique are normally lost due to their low amplitude. Especially, this is the case of diffracted echoes from planar misoriented defects and also from volumetric ones (by a process which involves mode conversion on the defect surface) The aim of this work is to carry out some experience in order to show how discriminate among different defect typologies and how to apply the better sizing procedure for each echo indication class depicted by S-scan image.

The experimentation has been carried out using the special test block sketched in figure 1 for what concern planar defects (produced by spark erosion), while for the volumetric ones, standard calibration blocks with SDH has been used

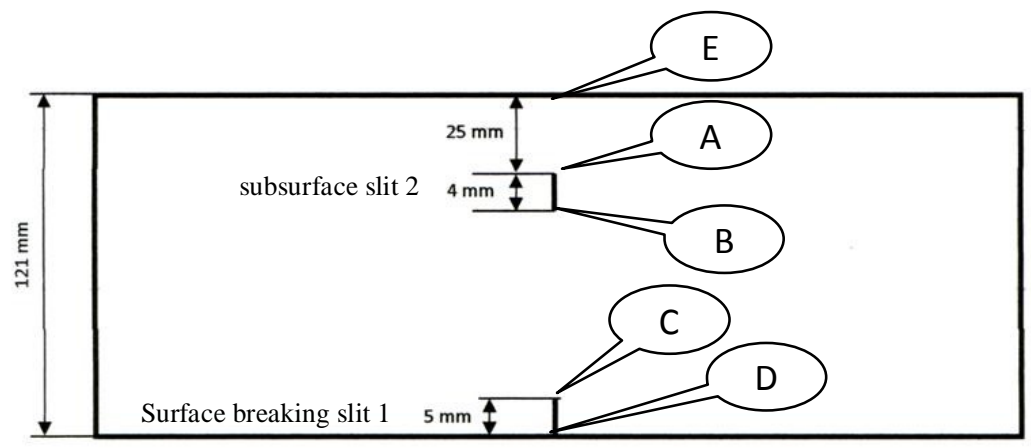

Figure 1: Sketch of the test block containing planar defects. The letters marks reflection(D,E)/diffraction( $A, B, C)$ points

\section{Diffraction from surface breaking slit}

Using the test block of figure 1, an inspection has been carried out from the opposite surface to that where is present the surface breaking slit 1 using two probe type, one working at $2.25 \mathrm{MHz}$ and the other at $5 \mathrm{MHz}$. The relevant sectorial scan images are reported, respectively, in figure 2 and 3 . In both figures, the stronger 


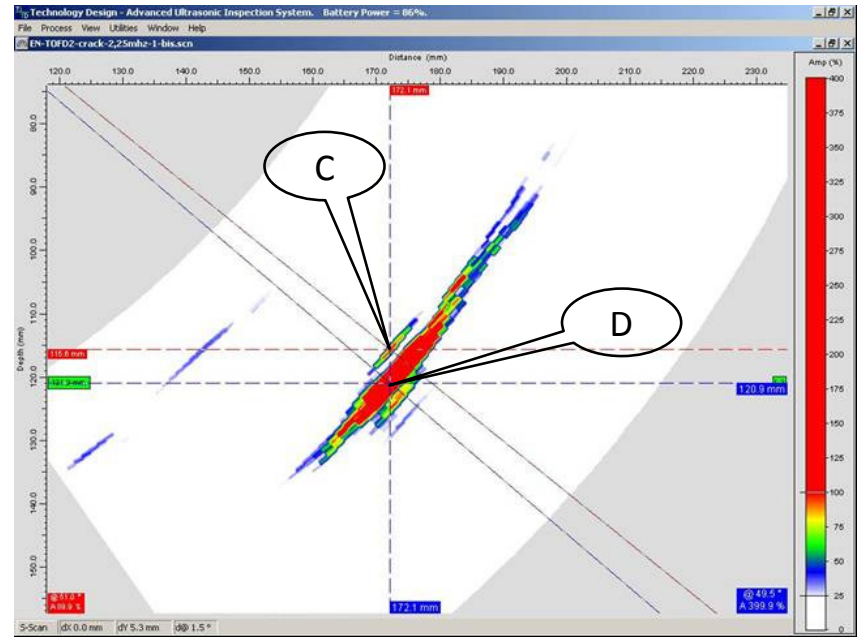

Figure 2: slit 1 Sectorial-scan view with $2.25 \mathrm{MHz}$ probe

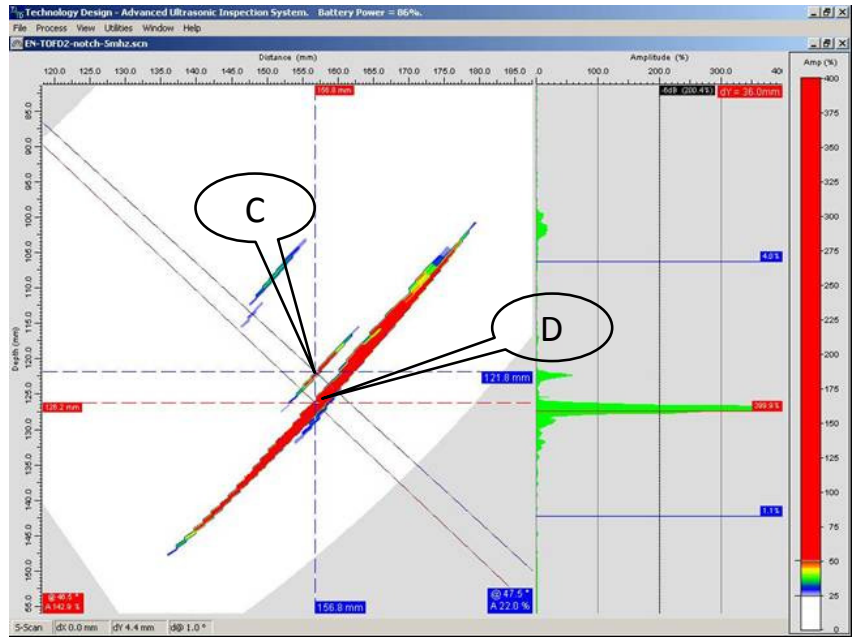

Figure 3: slit 1 Sectorial-scan view with $5 \mathrm{MHz}$ probe

echo is that generated by corner effect (D). Nevertheless is well visible, in both cases, the echo generated by the upper tip (C) diffraction and, thus, the sizing of surface breaking slit 1 height can be estimated simply measuring the distance of the tips from the bottom surface as shown in the figures by the dashed horizontal lines. Appling this procedure to figure 1, we obtain a value of $5.3 \mathrm{~mm}$ with an error of only $0.3 \mathrm{~mm}$, while from the figure 2 we obtain an estimated value of $4.4 \mathrm{~mm}$, again with an error of only $0.6 \mathrm{~mm}$. With respect to $2.25 \mathrm{MHz}$ probe, the $5 \mathrm{MHz}$ image, as expected, is better resolved. . The amplitude of echo diffracted by the notch tip, for the $2.25 \mathrm{MHz}$ probe, is about the $22 \%$ of DAC $(-13 \mathrm{~dB})$

\section{Diffraction from subsurface slit}

In this case the inspection has been carried out from the opposite surface to that where is present the surface breaking slit 1 with both probe at 2.25 and $5 \mathrm{MHz}$. The images shown figure 4 and 5 put in evidence the

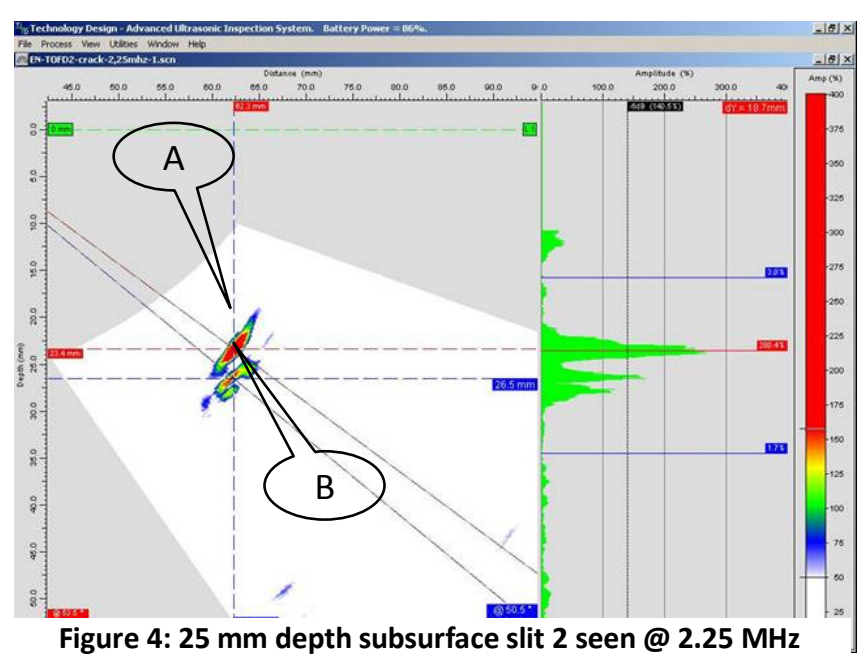

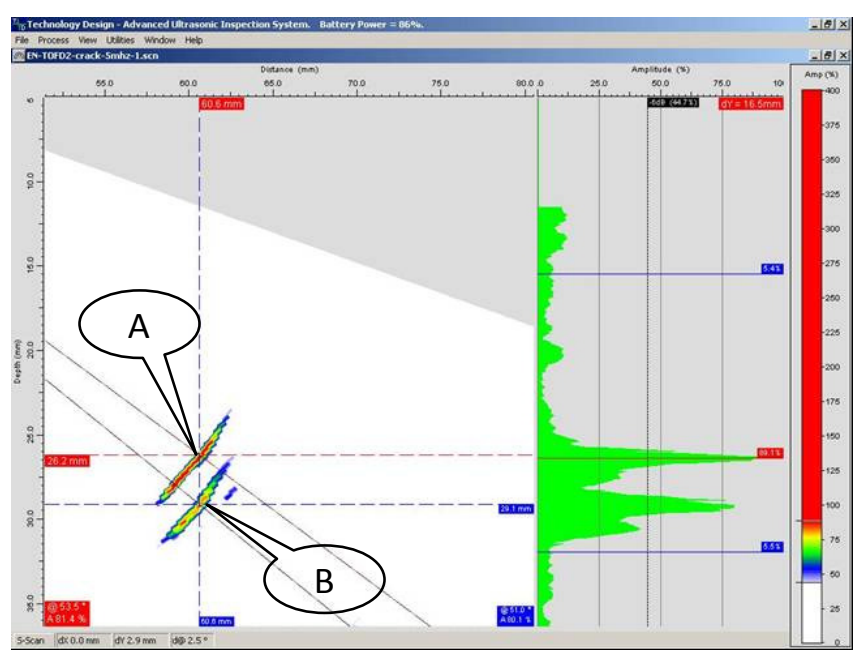

Figure 5: 25 mm depth subsurface slit 2 seen @ 5 MHz

diffracted echoes from upper (A) and lower tip (B) of the $25 \mathrm{~mm}$ depth subsurface crack depicted in figure 1. Estimation of defects height, as in the previous case, is done simply measuring the vertical distance between The images of the two tips as shown in the figures by the dashed lines. At $2.25 \mathrm{MHz}$ estimation of defect height gives the value of $3.1 \mathrm{~mm}$, which slightly under estimate the real one of $0.9 \mathrm{~mm}$; while at $5 \mathrm{MHz}$, the value is $2.9 \mathrm{~mm}$, which slightly under estimate the real one of $0.9 \mathrm{~mm}$. At $2.25 \mathrm{MHz}$ the amplitude of upper tip echo is $-6.6 \mathrm{~dB}$ DAC, while the ratio of the amplitude of lower tip echo with respect to that of upper tip is 0.53 . At $5 \mathrm{MHz}$ the amplitude of upper tip echo is $-17.7 \mathrm{~dB} \mathrm{DAC}$, while the ratio of the amplitude of lower tip echo with respect to that of upper tip is 0.84 . 


\section{Diffraction from subsurface slit near the back wall surface}

In this case the same subsurface defect of previous paragraph has been inspected from the surface where is present the surface breaking slit 1. Figure 6 and 7 shows the resultant images obtained with the 2.25 and 5 $\mathrm{MHz}$.

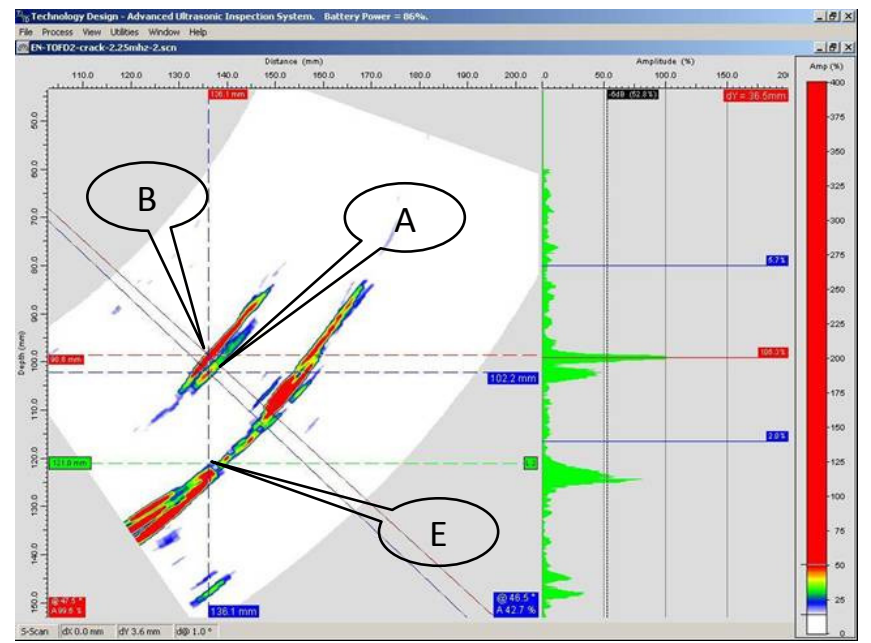

Figure 6: subsurface slit 2 seen @ $2.25 \mathrm{MHz}$

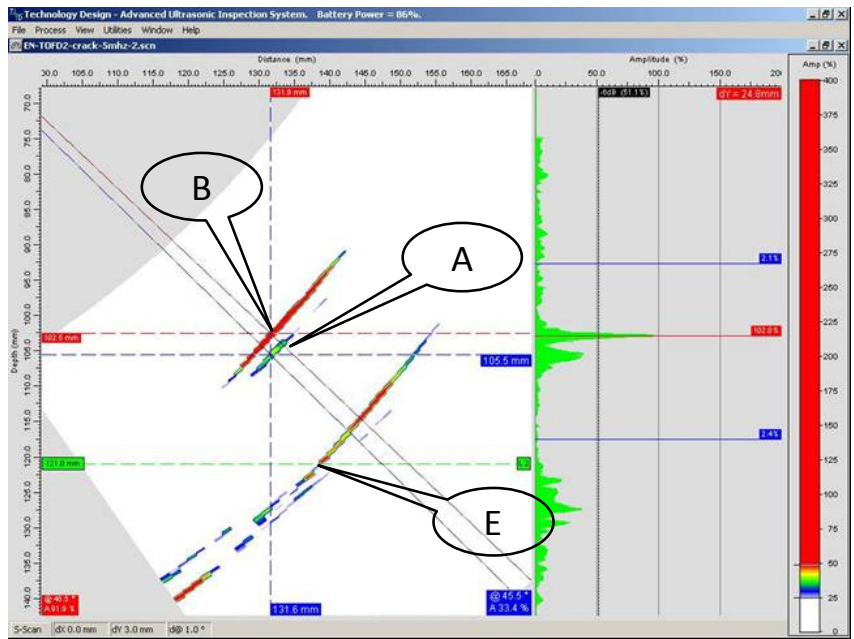

Figure 7: subsurface slit 2 seen @ $5 \mathrm{MHz}$

Images show diffracted echoes from upper (B) and lower tip (A) of the slit 2 and a stronger one due to partial reflection by corner effect $(\mathrm{E})$. At $2.25 \mathrm{MHz}$, the amplitude of upper tip echo is $-15.1 \mathrm{~dB} \mathrm{DAC}$, while the ratio of the amplitude of lower tip echo with respect to that of upper tip is 0.47 . Sizing carried out on the two echo basis gives the value of $3.6 \mathrm{~mm}$ which slightly under estimate the real one of $0.4 \mathrm{~mm}$. At $5 \mathrm{MHz}$, the amplitude of upper tip echo is $-16.6 \mathrm{~dB}$ DAC, while the ratio of the amplitude of lower tip echo with respect to that of upper tip is 0.39 . Sizing carried out on the two echo basis gives the value of $3.6 \mathrm{~mm}$ which slightly under estimate the real one of $0.4 \mathrm{~mm}$. With respect to $2.25 \mathrm{MHz}$ probe we still obtain a better resolved image but with worse sensitivity; in fact the maximum diffracted echo amplitude is equal to $14.8 \%$ DAC, which is, obviously, lower than threshold value of $20 \%$. As a general conclusion, comparing the performance of the $2.25 \mathrm{MHz}$ and the $5 \mathrm{MHz}$ probes it seems better to choose the $2.25 \mathrm{MHz}$ one. In fact, against a slightly worse resolution we have a better sensitivity.

\section{Diffraction from volumetric defects}

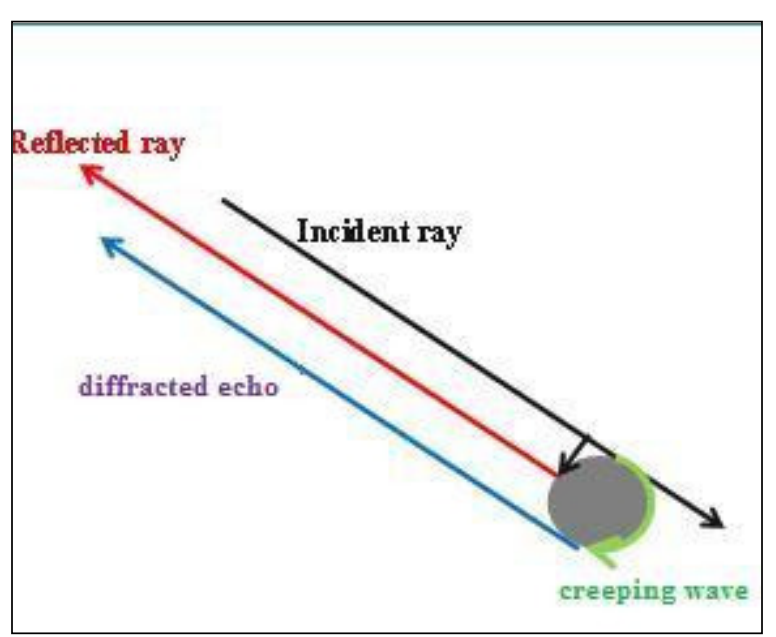

Figure 8: sketch illustrating the complex behavior of volumetric diffraction
Figure 8 shows the complex mechanism of volumetric diffraction. The incident rays are partially back specular reflected; but some of them induce a creeping wave on the volumetric defect surface. The creeping wave, while travelling around the volumetric defect, reemits bulk waves and some of them are received by the probe which, after the main specular reflected echoes, will display a small amplitude secondary echo (improperly called diffracted echo).

The distance $\mathrm{L}$ between the main echo and this secondary echo depends clearly by the defect diameter D through the empirical formula:

$$
\mathrm{L}=1.39 * \mathrm{D}
$$

On a Sectorial-scan image, such satellite echo is always located after the main image and along the beam axis; this behavior is useful because can help to distinguish volumetric defects from planar ones. 
Figure 9 shows the images of the three $3 \mathrm{~mm}$ SDH hole of an ASME calibration block. All the SDH images, which are seen at different angle, show the secondary/diffracted echoes and confirm that such secondary images, as stated before, are placed always on the beam axis, that is, the line joining transducer - defect.

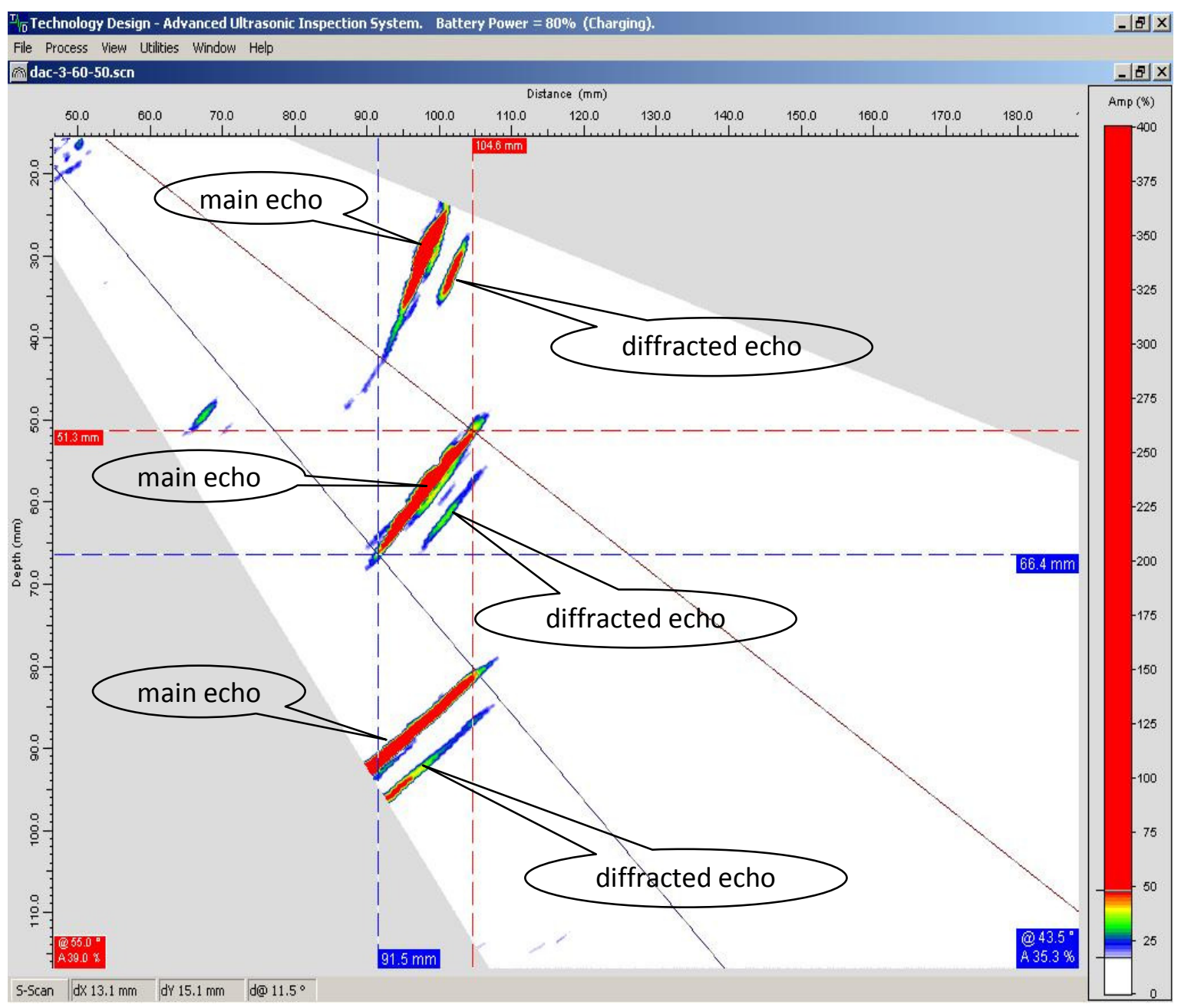

In the case of figure 9, the distance $\mathrm{L}$, between main and secondary echoes, are about $4.3 \mathrm{~mm}$, and, using the previous formula, we obtain for SDH diameter the value of $3.2 \mathrm{~mm}$ which overestimate the real value of only $0.2 \mathrm{~mm}$. 


\section{Discrimination between planar and volumetric in presence of diffracted echoes}

Figure 10 shows a comparison between the characteristics of reflection / diffraction relevant to

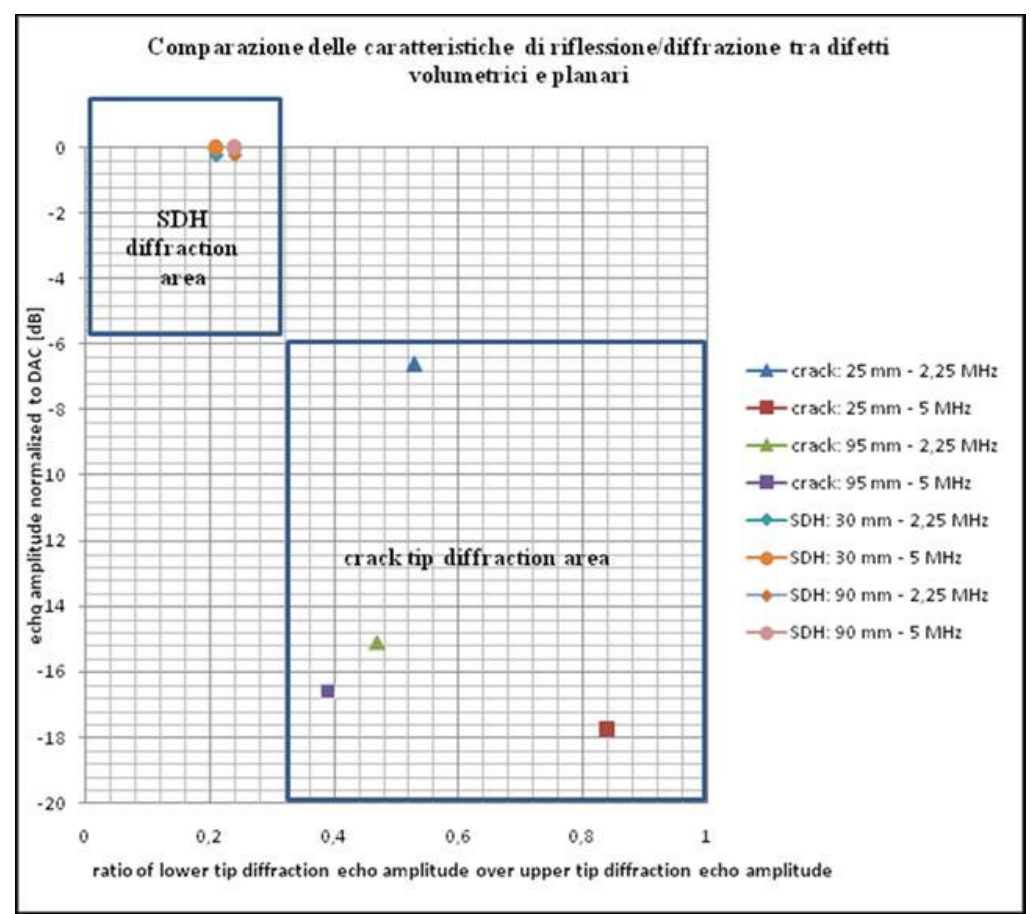

Figure 10: difference of echo parameters for planar and volumetric defects subsurface crack and those of the equivalent $3 \mathrm{~mm}$ SDH hole used for calibration. Also for the SDH we can observe a diffracted echo which follows the main reflection. Thus it is interesting to determine a suitable criteria which can help to correctly discriminate the defect typology. In particular, the graph shows the DAC echo amplitude values against the ratio between the lower and upper diffracted echo amplitude. As we can observe, the amplitude of volumetric defect is, obviously, higher than that of diffracted echo while the ratio relevant to volumetric defect is lower than half the ratio relevant to crack like defect. Thus We can draw two rectangle which enclose two different and disjoined region each of one pertains to a single defect typology

\section{Clustered indications}

Figure 11 shows an example of a cluster sizing. The cluster is composed of four $2 \mathrm{~mm} \mathrm{SDH}$ holes vertically aligned with a $1 \mathrm{~mm}$ separation for a total height of 11 $\mathrm{mm}$. The image is obtained with a 32 elements probe for shear wave working at $2.25 \mathrm{MHz}$. The apparent height is measured putting the saturation red color threshold at -12 $\mathrm{dB}$ with respect to the DAC and, then, determining the smallest rectangle which enclose the red region. In this case we obtain a value of $14.2 \mathrm{~mm}$ which can be corrected subtracting one fourth of the vertical resolution at the depth and angle view of the cluster. In this case we obtain the more realistic

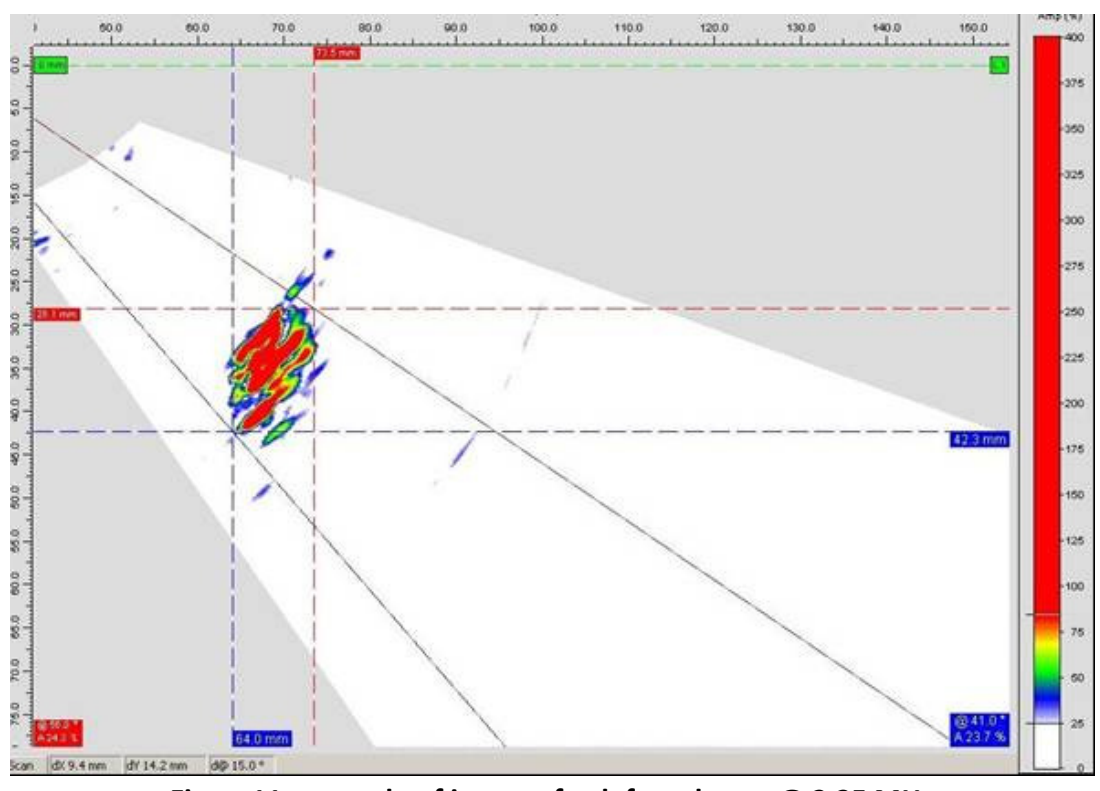

Figure11: example of image of a defect cluster @ 2.25 MHz value of $11.8 \mathrm{~mm}$. 


\section{Sizing procedure in case of single indications}

The most critical case for sizing is represented by the so called single indications which are typically generated by defects whose height is small compared with ultrasonic wavelength so that eventually diffracted echoes collapse together in a single echo. Other cases are represented by defects of complex geometry for which one of the diffracted/secondary echoes disappears. The typical parameter we can determine is the defect apparent height measured as the case of cluster at $-12 \mathrm{db}$ of DAC. But a good sizing procedure require a comparison of such apparent eight $\mathbf{h}_{\mathbf{i}}$ with the apparent height of some reference SDH reflectors.

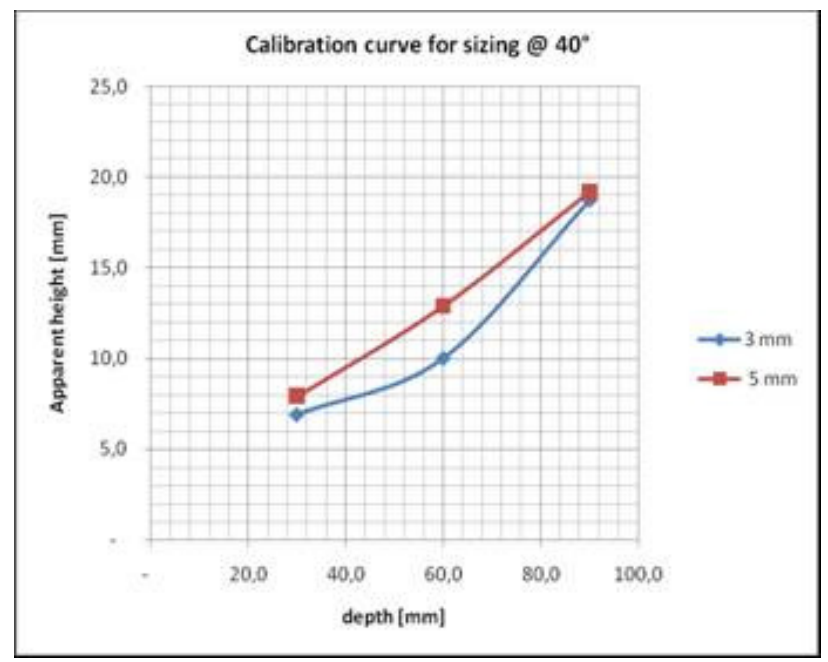

Figure 12: 3 and $5 \mathrm{~mm}$ SDH apparent height against depth @ $40 \mathrm{deg}$.

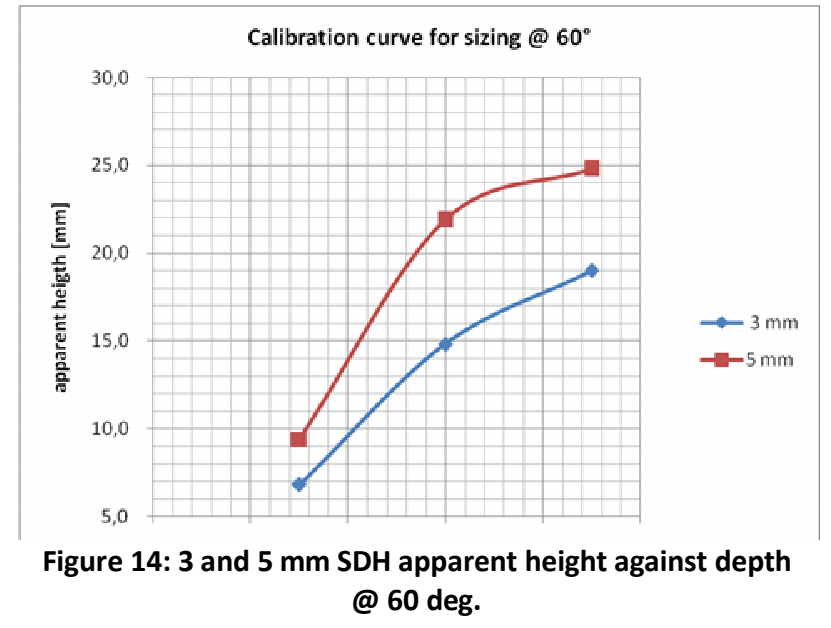

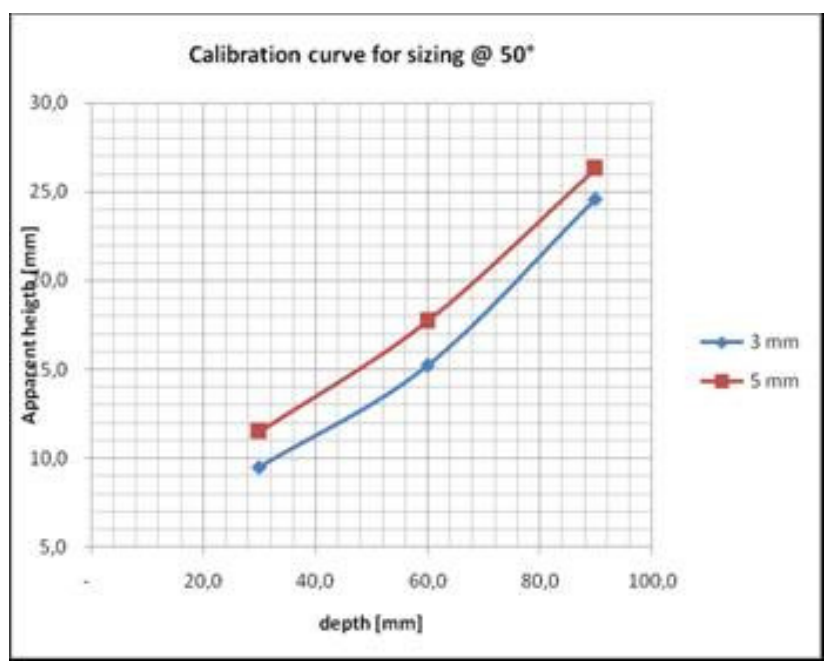

Figure 13: 3 and $5 \mathrm{~mm}$ SDH apparent height against depth @ 50 deg.

Thus we need suitable calibration curves as, for example, those of figure $12,13,14$, which report the graph of apparent height $\mathbf{h}_{\mathbf{3}}$ and $\mathbf{h}_{\mathbf{5}}$, relevant to $3 \mathrm{~mm}$ and $5 \mathrm{~mm} \mathrm{SDH}$ reflectors, against depth and seen at different angles.

One calibration curve, among those of previous figures, shall be selected as the one whose angle is the nearest to the defect indication view angle. On this curve the apparent height $\mathrm{h}_{3}$ and $\mathrm{h}_{5}$, relevant to reference reflectors of 3 and $5 \mathrm{~mm}$, shall be read at the defect indication depth. Thus the following step shall be done:

a) if $\mathbf{h}_{\mathbf{i}} \leq \mathbf{h}_{\mathbf{3}}$, then the real defect height $\mathrm{H}$ shall be computed using the following formula:

$$
\mathbf{H}=\mathbf{3} * \mathbf{h}_{\mathrm{i}} / \mathbf{h}_{\mathbf{3}}
$$

This formula is equivalent to an interpolation on the straight line passing from the origin and from the point $\left(3, h_{3}\right)$.

b) if $\mathbf{h}_{\mathbf{i}}>\mathbf{h}_{\mathbf{3}}$, then the real defect height $\mathrm{H}$ shall be computed using the following formula:

$$
\mathbf{H}=\mathbf{3}+\left(\mathbf{h i}-\mathbf{h}_{3}\right) *(\mathbf{5 - 3}) /\left(\mathbf{h}_{\mathbf{5}}-\mathbf{h}_{\mathbf{3}}\right)
$$

This formula is equivalent to an interpolation on the straight line passing from the point $\left(3, \mathrm{~h}_{3}\right)$ and from the point $\left(5, \mathrm{~h}_{5}\right)$. 


\section{Conclusions}

We have shown that, using phased array technology, it is possible to discriminate among different image patterns, due to different defect typology, and that for each class of echoes indications a suitable sizing procedure for defect height estimation can gives good results.

\section{References}

[1] Sharp R. S., "Research Techniques in Non Destructive Testing", Vol. IV, 1980

[2] Malcolm, J. Crocker, "Encyclopedia of Acoustics", Vol. One, 1997

[3] ASME Code Sec. V, art. 4, Code Case 2235.9. Use of Ultrasonic Examination in Lieu of Radiography.

[4] Ichiro Komura, Taiji Hirasawa, Satoshi Nagai (Toshiba),Jun-ichi Takabayashi (Keihin), Katsuhiko

Naruse (Isogo Nuclear) "Crack Detection and Sizing Technique by Ultrasonic and Electromagnetic

Methods", Proceedings of the $25^{\text {th }}$ MPA Seminar, MPA Stuttgart, Germany, October 7 - 8, 1999

[5] R/D Tech, "Introduction to Phased Array Ultrasonic Technology Applications: R/D Tech Guideline",

Quebec City, Canada, R/D Tech, 2004.

[6] Lafontaine, G. and F. Cancre, "Potential of Ultrasonic Phased Arrays for Faster, Better and Cheaper Inspections," NDT.net, Vol. 5, No. 10, October 2000, <www.ndt.net/article/v05n10/lafont2/lafont2.htm〉.

[7] Nardoni G., "CB-Scan Technique with Phased Array Probe: Sizing procedures using diffracted echoes", Conference on ASME Code Case 2235.9: From acceptance criteria based on amplitude of signal to acceptance criteria based on size, Moscow, 2008 